Sādhanā, Vol. 19, Part 1, February 1994, pp. 93-111. (C) Printed in India.

\title{
A prototype expert system for interpretation of remote sensing image data
}

\section{CHANDRA SEKHARA SARMA ${ }^{1}$ and V V S SARMA ${ }^{2}$}

${ }^{1}$ ISRO Computer Office, Indian Space Research Organisation HQ, Bangalore 560094, India

${ }^{2}$ Department of Computer Science and Automation, Indian Institute of Science, Bangalore 560012 , India

E-mail: ${ }^{1}$ lcs@isro.ernet.in; ${ }^{2}$ vvs@csa.iisc.ernet.in

\begin{abstract}
Automated image interpretation systems of remotely sensed images are of great help in the present scenario of growing applications. In this paper, we have critically studied visual interpretation processes for urban land cover and land use information. It is observed that the core activity of interpretation can be described as plausible combinations of pieces of evidential information from various sources such as images, collateral data, experiential knowledge and pragmatics. Interpretation keys for the interpretation of standard false colour composites are considered to be tone/colour, pattern, texture, size, shape, association, relief and season. These interpretation keys encompass the spectral, spatial and temporal knowledge required for image interpretation. Our focus is on a knowledge-based approach for interpretation of standard false colour composites (FCC). Basic information required for a knowledge-based approach is of four types viz., spectral, spatial, temporal and heuristic. Generic classes and subclasses of image objects are identified for the land use/land cover theme. Logical image objects are conceptualised as region/ area, line and point objects. An object-oriented approach for the representation of spectral and spatial knowledge has been adopted. Heuristic information is stored in rules. The Dempster-Shafer theory of evidence is used to combine evidence from various interpretation keys for identification of generic class and subclass of a logical image object. Analysis of some Indian Remote Sensing Satellite images has been done using various basic probability assignments in combination with learning. Explanation facility is provided by tracing the rules fired in the sequence.
\end{abstract}

Keywords. Remote sensing; image interpretation; false colour composite; interpretation keys; expert systems; domain objects; Dempster-Shafer theory; uncertainty handling.

\section{Introduction}

The need for automated image interpretation systems with expert-level performance has been long felt. Although the intent of computer-assisted digital image classification 
is to generate thematic maps using more quantitative methods, visual interpretation is still indispensable for attaining expert-level performance. To a large extent, this is due to the inadequacies of digital classification such as lack of temporal, spatial and neighbourhood knowledge. The need for expert-level performance in image interpretation has brought in a paradigm shift from domain independent statistical methods to domain specific knowledge-based techniques (Argialas 1990). The core activity of interpretation can be described as a plausible combination of pieces of evidential information from various sources such as characteristic features of image objects, domain-specific knowledge, collateral data and pragmatics. This activity is more in the nature of explorative and qualitative reasoning in the line of artificial intelligence (AI) and expert systems (ES). AI and ES techniques have contributed powerful and flexible methodologies to represent domain-specific knowledge and heuristic problem-solving knowledge in the domain of image interpretation which are often declarative in nature. While many knowledge-based methodologies combining AI, pattern recognition and image analysis have been proposed by quite a few researchers in the recent past (Wang \& Newkirk 1988; Schowengerdt \& Wang 1989), there are hardly any systems which consider knowledge from spectral, spatial and temporal domains together for interpreting an image.

We have developed a prototype expert system for the interpretation of false colour composites (FCC) of IRS-1A (Indian Remote-Sensing Satellite) for land use/land cover categorization theme, using GC LISP on a personal computer. Various logical components of this system are given in figure 1. The visual interpretation key developed by the National Natural Resources Management Systems (NNRMS) Office, Department of Space, has been used as a basis for our knowledge-based approach, which covers the required knowledge from all the three domains mentioned before. Image interpretation activity is viewed as a data fusion activity in which the sources of evidence are features such as colour, texture, pattern, size, shape, association, relief and season. Knowledge is represented in property lists of GC LISP in the form of objects and rules. Knowledge organization is hierarchical (two-level) and control sequence is sequential. Reasoning for identification of logical image objects is done using Dempster's

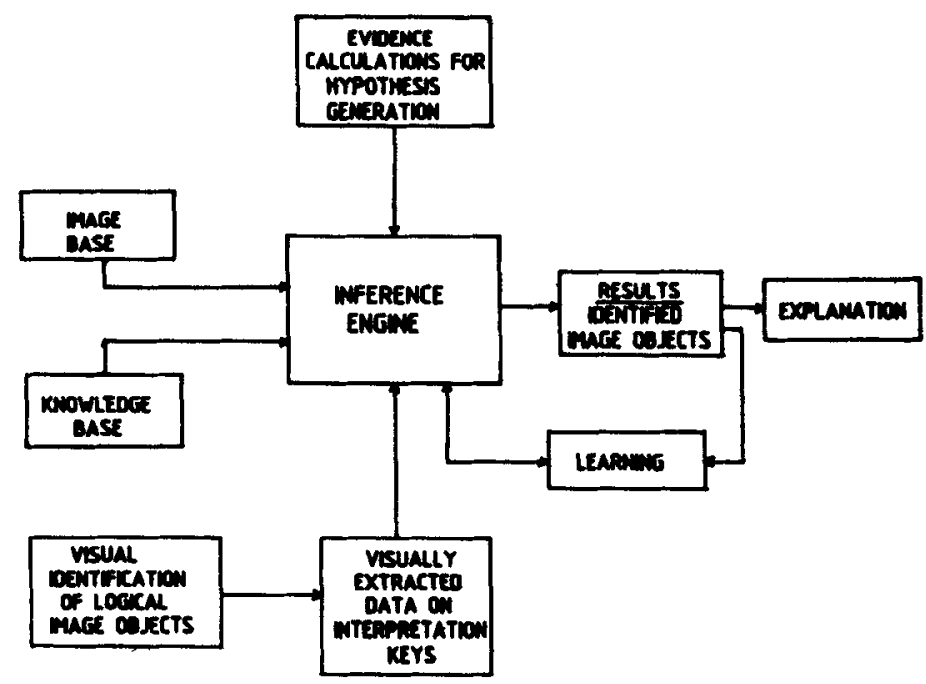

Figure 1. Various logical components of the expert system. 
combination rule for combining evidential information from the various features mentioned above.

\section{Formulation of knowledge base}

\subsection{Knowledge elicitation}

For knowledge elicitation, we have conducted focused and structured interviews with experts available at the NNRMS office based on the interpretation key developed by a team of experts in the theme of land use/land cover categorization. Part of the interpretation key is shown in table 1 . Imprecision and vagueness in the description of feature values is recognized and the experts agree with it as it is because of inherent fuzziness in human expression. Knowledge was elicited about variation in weightages to be allocated to feature values based on domain, rules for interpretation and conflict resolution, in the case of different results being obtained with the same subset of feature values. The term 'interpretation key' is used in the sense of 'feature' in further discussions.

\subsection{Types of the knowledge}

We identify the types of knowledge and data in the domain of image interpretation and the relevant goals, as shown in table 2 . This analysis provides support in designing a knowledge-based system, in deciding the choice of the knowledge-representation and uncertainty-handling schemes and in performance analysis (Hayes-Roth 1989).

\subsection{Uncertainty handling}

Image interpretation involves decreasing the local ambiguity and merging the pieces of knowledge (associated with the interpretation keys) into a unique interpretation. The disambiguation process calls for handling uncertainty in the domain of image interpretation. We choose to accept the confidence factors provided by the user, which are representatives of the user belief in expressing the values of the corresponding features of an image object. Reasoning is done to identify image objects based on the feature values and associated certainty factors.

\subsection{Steps involved in the system design}

To model and implement a knowledge-based system for interpretation of satellite imagery, four levels are identified. They are the conceptual, representation, reasoning and idealization levels as shown in figure 2.

In our system spectral, spatial and temporal knowledge embedded in the interpretation keys together generate a hypothesis based on image-domain, scene-domain specifications (shown in figure 3 ) and on the user's confidence in the description of the feature values. This hypothesis may suggest a subset of identification names, which is further refined using real-world knowledge and heuristics to label an image object. 
Table 1. Land use/land cover interpretation key using satellite remote sensing imagery.

\begin{tabular}{|c|c|c|c|c|c|c|}
\hline $\begin{array}{l}\text { St. } \\
\text { No. }\end{array}$ & $\begin{array}{l}\text { Land use/ } \\
\text { land cover } \\
\text { category }\end{array}$ & Tone/colour & Size & Shape & Texture & Pattern \\
\hline 01 & Built-up land & $\begin{array}{l}\text { Dark bluish green } \\
\text { in the core and } \\
\text { bluish on the peri- } \\
\text { phery }\end{array}$ & $\begin{array}{l}\text { Small } \\
\text { to big }\end{array}$ & $\begin{array}{l}\text { Irregular \& } \\
\text { discontinuous }\end{array}$ & $\begin{array}{l}\text { Coarse \& } \\
\text { mottled }\end{array}$ & $\begin{array}{l}\text { Clustered to } \\
\text { scattered \& } \\
\text { non-contiguous }\end{array}$ \\
\hline 02 & Transportation & $\begin{array}{l}\text { Very dark to } \\
\text { dark bluish green, } \\
\text { light yellow for } \\
\text { minor roads, red } \\
\text { if vegetation } \\
\text { along the road }\end{array}$ & $\begin{array}{l}\text { Small in } \\
\text { width for } \\
\text { roads and } \\
\text { narrow for } \\
\text { rail }\end{array}$ & $\begin{array}{l}\text { Regular with } \\
\text { straight/sharp } \\
\text { and smooth } \\
\text { curves }\end{array}$ & $\begin{array}{l}\text { Smooth to } \\
\text { fine }\end{array}$ & $\begin{array}{l}\text { Linear to } \\
\text { sinuous } \& \\
\text { contiguous }\end{array}$ \\
\hline 03 & Crop land & $\begin{array}{l}\text { Bright red } \\
\text { to red }\end{array}$ & $\begin{array}{l}\text { Varying in } \\
\text { size }\end{array}$ & $\begin{array}{l}\text { Regular to } \\
\text { irregular }\end{array}$ & $\begin{array}{l}\text { Medium to } \\
\text { smooth }\end{array}$ & $\begin{array}{l}\text { Contiguous to } \\
\text { non-contiguous }\end{array}$ \\
\hline 04 & Fallow land & $\begin{array}{l}\text { Yellow to } \\
\text { greenish blue } \\
\text { (depending on soil } \\
\text { type and moisture) }\end{array}$ & $\begin{array}{l}\text { Small to } \\
\text { large }\end{array}$ & $\begin{array}{l}\text { Regular to } \\
\text { irregular }\end{array}$ & $\begin{array}{l}\text { Medium to } \\
\text { smooth }\end{array}$ & $\begin{array}{l}\text { Contiguous to } \\
\text { non-contiguous }\end{array}$ \\
\hline 05 & $\begin{array}{l}\text { Plantation } \\
\text { (agriculture) }\end{array}$ & $\begin{array}{l}\text { Dark red to } \\
\text { red }\end{array}$ & $\begin{array}{l}\text { Small to } \\
\text { medium }\end{array}$ & $\begin{array}{l}\text { Regular with } \\
\text { sharp edges }\end{array}$ & $\begin{array}{l}\text { Coarse to } \\
\text { medium }\end{array}$ & $\begin{array}{l}\text { Dispersed } \\
\text { contiguous }\end{array}$ \\
\hline 06 & $\begin{array}{l}\text { Evergreen/ } \\
\text { Semi-ever- } \\
\text { green forest }\end{array}$ & $\begin{array}{l}\text { Bright red to } \\
\text { dark red }\end{array}$ & $\begin{array}{l}\text { Varying in } \\
\text { size }\end{array}$ & $\begin{array}{l}\text { Irregular, } \\
\text { discontinuous }\end{array}$ & $\begin{array}{l}\text { Smooth to } \\
\text { medium } \\
\text { depending } \\
\text { on crown } \\
\text { density }\end{array}$ & $\begin{array}{l}\text { Contiguous to } \\
\text { non-contiguous }\end{array}$ \\
\hline 07 & $\begin{array}{l}\text { Deciduous } \\
\text { forest }\end{array}$ & $\begin{array}{l}\text { Dark red } \\
\text { to red }\end{array}$ & $\begin{array}{l}\text { Varying in } \\
\text { size }\end{array}$ & $\begin{array}{l}\text { Irregular, } \\
\text { discontinuous }\end{array}$ & $\begin{array}{l}\text { Smooth to } \\
\text { medium } \\
\text { depending } \\
\text { on crown } \\
\text { density }\end{array}$ & $\begin{array}{l}\text { Contiguous to } \\
\text { non-contiguous }\end{array}$ \\
\hline
\end{tabular}




\begin{tabular}{|c|c|c|c|}
\hline Location & Association & Season & Remarks \\
\hline $\begin{array}{l}\text { Plains, plateaus, } \\
\text { on hill slopes, } \\
\text { deserts, water- } \\
\text { front, road, rail, } \\
\text { canal etc. }\end{array}$ & $\begin{array}{l}\text { Surrounded by agri- } \\
\text { cultural lands, forest } \\
\text { cover, wastelands, } \\
\text { network of rivers, } \\
\text { roads, and rail etc. }\end{array}$ & $\begin{array}{l}\text { October } \\
\text { to } \\
\text { March }\end{array}$ & $\begin{array}{l}\text { Built-up land can be of big or small size } \\
\text { settlements, industrial structures, buildings or any } \\
\text { other artifact, physical spread or sprawl along with } \\
\text { density of transport network are useful surrogates } \\
\text { to classify it as urban or rural. Perceptible' land } \\
\text { transformation can be noticed around built-up land }\end{array}$ \\
\hline $\begin{array}{l}\text { On all types of } \\
\text { terrain, across } \\
\text { water bodies, } \\
\text { agricultural lands } \\
\text { connecting settle- } \\
\text { ments }\end{array}$ & $\begin{array}{l}\text { Settlement nodes, } \\
\text { amidst and around } \\
\text { built-up developed } \\
\text { areas etc. }\end{array}$ & $\begin{array}{l}\text { October } \\
\text { to } \\
\text { March }\end{array}$ & $\begin{array}{l}\text { Provides connectivity linkages between } \\
\text { settlements and accelerates development. Road, } \\
\text { rail and canal vary in dimension and importance. } \\
\text { Can be mapped in detail using infrared bands } \\
\text { and higher spatial resolution data. Forms part of } \\
\text { non-agricultural use }\end{array}$ \\
\hline $\begin{array}{l}\text { Plains, hill slopes, } \\
\text { valleys, cultivable } \\
\text { wastelands etc. }\end{array}$ & $\begin{array}{l}\text { Amidst irrigated } \\
\text { (canal, tank, well etc.) } \\
\text { and unirrigated } \\
\text { (rainfed/dry farming) } \\
\text { arable lands, proxi- } \\
\text { mity to rivers/streams } \\
\text { etc. }\end{array}$ & $\begin{array}{l}\text { June to } \\
\text { September } \\
\text { and } \\
\text { October } \\
\text { to } \\
\text { April }\end{array}$ & $\begin{array}{l}\text { Consists of different crops grown in } \\
\text { different seasons under different farming and } \\
\text { land-tenural systems. Mixed and multiple } \\
\text { cropping patterns generate mixed spectral } \\
\text { response on the images }\end{array}$ \\
\hline $\begin{array}{l}\text { Plains, valleys } \\
\text { uplands etc. }\end{array}$ & $\begin{array}{l}\text { Amidst crop land } \\
\text { as harvested agri- } \\
\text { cultural fields etc. }\end{array}$ & $\begin{array}{l}\text { January } \\
\text { to } \\
\text { December }\end{array}$ & $\begin{array}{l}\text { Consists of different arable lands left } \\
\text { uncultivated as seasonal/temporary fallows for } \\
\text { less than a year and as permanent fallows up to } 5 \\
\text { years or more because of diverse reasons. Fallow } \\
\text { land devoid of vegetation, accelerates erosion }\end{array}$ \\
\hline $\begin{array}{l}\text { Plains, foot hills } \\
\text { and uplands }\end{array}$ & $\begin{array}{l}\text { Dry lands or un- } \\
\text { irrigated lands, } \\
\text { uplands occasionally } \\
\text { amidst crop land, } \\
\text { proximity to rivers } \\
\text { and on gentle hill } \\
\text { slopes }\end{array}$ & $\begin{array}{l}\text { January } \\
\text { to } \\
\text { December }\end{array}$ & $\begin{array}{l}\text { Agricultural plantations consist of a variety of } \\
\text { trees, orchards and groves. These occur } \\
\text { throughout the year and are seen very } \\
\text { prominently on the imagery. Those occurring } \\
\text { in the forest areas (but outside the notified } \\
\text { forest areas) are also treated as plantations like } \\
\text { coffee, tea, arecanut etc. }\end{array}$ \\
\hline $\begin{array}{l}\text { High relief } \\
\text { mountain/hill tops } \\
\text { and slopes and } \\
\text { within notified } \\
\text { areas }\end{array}$ & $\begin{array}{l}\text { High relief/slopes } \\
\text { exposed to very } \\
\text { heavy rainfall } \\
\text { zones. }\end{array}$ & $\begin{array}{l}\text { January } \\
\text { to } \\
\text { December }\end{array}$ & $\begin{array}{l}\text { These are closed ( } 40 \% \text { tree cover) or high } \\
\text { density forest cover of conifers and other } \\
\text { broad leaved forest trees. These coincide with the } \\
\text { zones of high rainfall and relief. They provide } \\
\text { shelter to wildlife and livestock. They influence the } \\
\text { climate and water regime and protect the environment }\end{array}$ \\
\hline $\begin{array}{l}\text { Medium relief } \\
\text { mountains/hill } \\
\text { slopes and within } \\
\text { notified areas }\end{array}$ & $\begin{array}{l}\text { Different forest } \\
\text { types/sub-types of } \\
\text { species which shed } \\
\text { leaves }\end{array}$ & $\begin{array}{l}\text { January } \\
\text { to } \\
\text { April }\end{array}$ & $\begin{array}{l}\text { These are broad-leaved tropical forests which } \\
\text { seasonally shed their leaves annually. Dry forest } \\
\text { trees are subject to wild forest fires particularly } \\
\text { during summer/autumn. These occur on the lower } \\
\text { elevations and slopes rather than in the evergreen/semi- } \\
\text { evergreen forests. }\end{array}$ \\
\hline
\end{tabular}


Table 2. Types of knowledge and data in image interpretation domain and relevant goals.

\begin{tabular}{ll}
\hline Type & \multicolumn{1}{c}{ Examples } \\
\hline $\begin{array}{l}\text { Objects } \\
\text { Domain objects }\end{array}$ & $\begin{array}{l}\text { Road, rail, canal; plantation, tank/reservoir, settlements, } \\
\text { industrial complexes, ships, tanks } \\
\text { Line objects, area objects, point objects }\end{array}$ \\
Scene objects & $\begin{array}{l}\text { Rivers do not cross each other } \\
\text { Red colour indicates 'vegetation' } \\
\text { If the scene is urban-land and colour is white and shape is } \\
\text { circular then the object is stadium }\end{array}$ \\
$\begin{array}{l}\text { Facts } \\
\text { Defaults }\end{array}$ & $\begin{array}{l}\text { Assume black colour indicates a water body in the first instance } \\
\text { Factual rule }\end{array}$ \\
$\begin{array}{l}\text { Streams are with unstructured pattern and with 'somewhat } \\
\text { narrow' starting and 'rather wide' ending } \\
\text { If the texture of vegetation is 'smooth to medium' then it may be } \\
\text { Fuzzy facts }\end{array}$ & $\begin{array}{l}\text { crop land } \\
\text { Fuzzy rule }\end{array}$
\end{tabular}

Domain structures

Elementary structures

Network structures

Group structures

Point, line and area

Drainage patterns

Industrial sheds with housing colonies

Prerequisites (data and data processing)

Spectral clarity

Collateral material

Preference

Enhancement, removal of noise in the pixels of image

Ground truth, toposheets, aerial photographs, geographic information system

FCC is preferred to $B / w$ image for land cover categorization

Problem-solving knowledge

Knowledge representation

Meta-knowledge

Heuristic meta-rule

Combination of evidence

Uncertainty handling

Conflict resolution

Pixel oriented/vector representation/object oriented

Examine line objects first for geological application; examine area objects first for land cover/land use applications

Fire the rule with maximum confidence first

Additive, non-additive, ad-hoc

Certainty factors, fuzzy calculus, belief measures

Interdependence of objects for recognizing 'association'

Goals

Civilian

Monitoring man-land ratio estimates, water resource allocation etc.

Military

Troop movement observation, approachability and formation of regiments etc.

\subsection{Knowledge -representation}

By and large, complex problems become tractable if one chooses the right level of abstraction, i.e. the set of appropriate terms in which to think about the domain. As an alternate approach to the image data base management systems which are found not suitable to handle feature-oriented image object knowledge, we conceptualize detectable image objects as point, line and region/area objects (Sarma \& Sarma 1990) and adapt object-oriented approach for knowledge representation of image objects. Generic classes and corresponding subclasses in the domain of land use/land cover categorization are identified and two sample classes are shown in table 3 . 


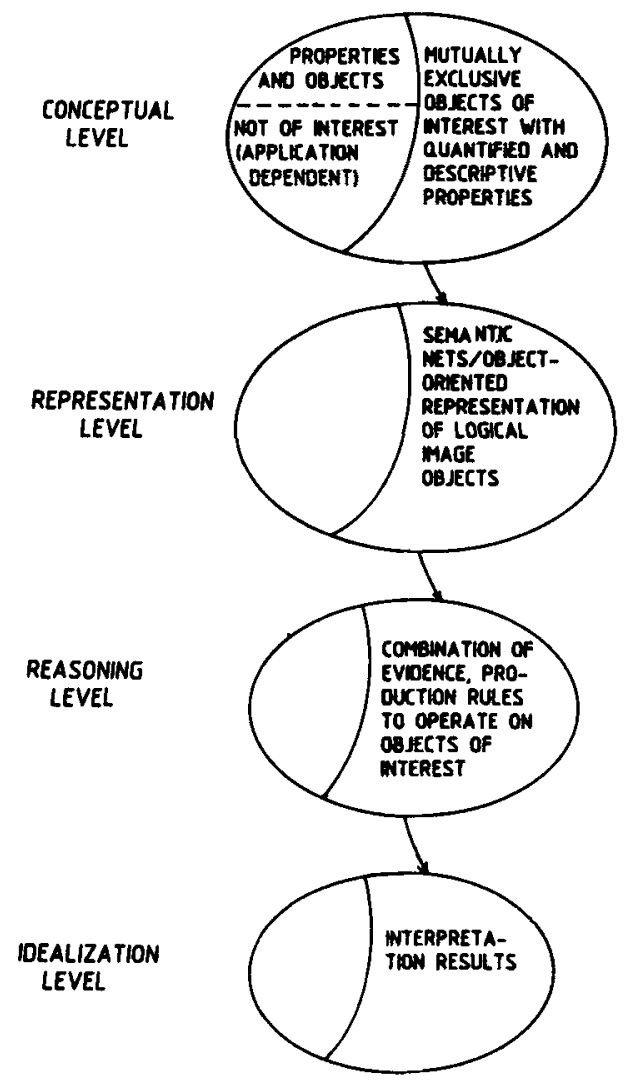

Figure 2. Steps involved in knowledge-based interpretation system design.

The class-subclass relation in a region object is represented using property lists in GC LISP as shown below.

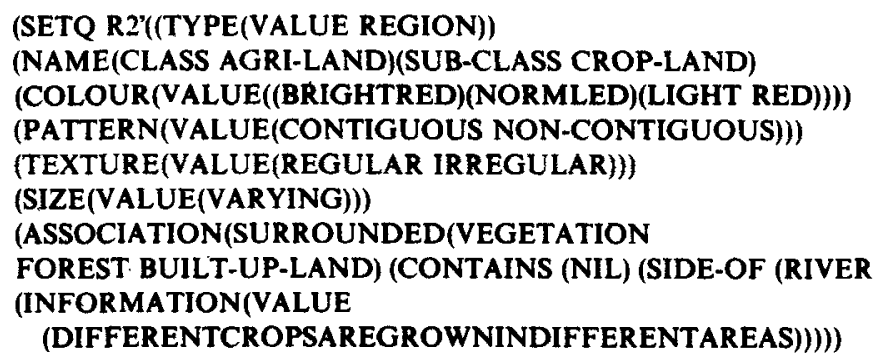

Table 3. Sample generic classes and corresponding subclasses in land use/land cover categorization.

\begin{tabular}{lc}
\hline Generic class & Subclass \\
\hline Vegetation & Crop land \\
& Plantation \\
& Forestry \\
Built-up land & Settlements \\
& Urban/rural \\
& Industrial \\
\hline
\end{tabular}


(a)

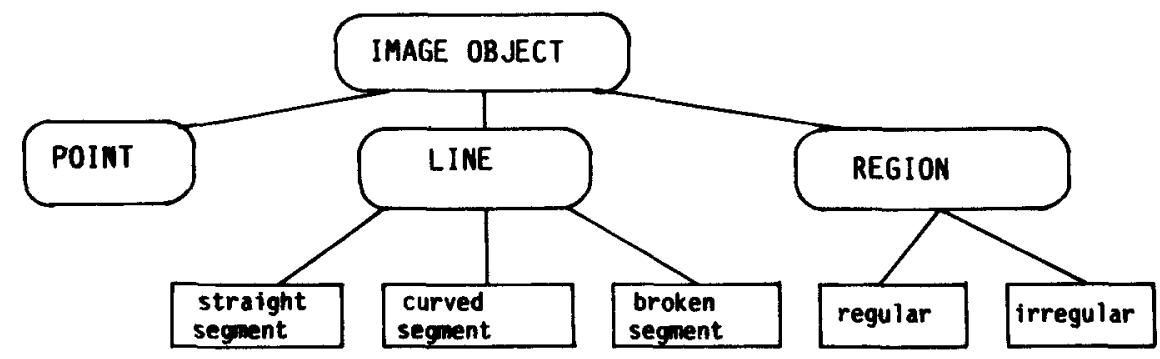

(b)

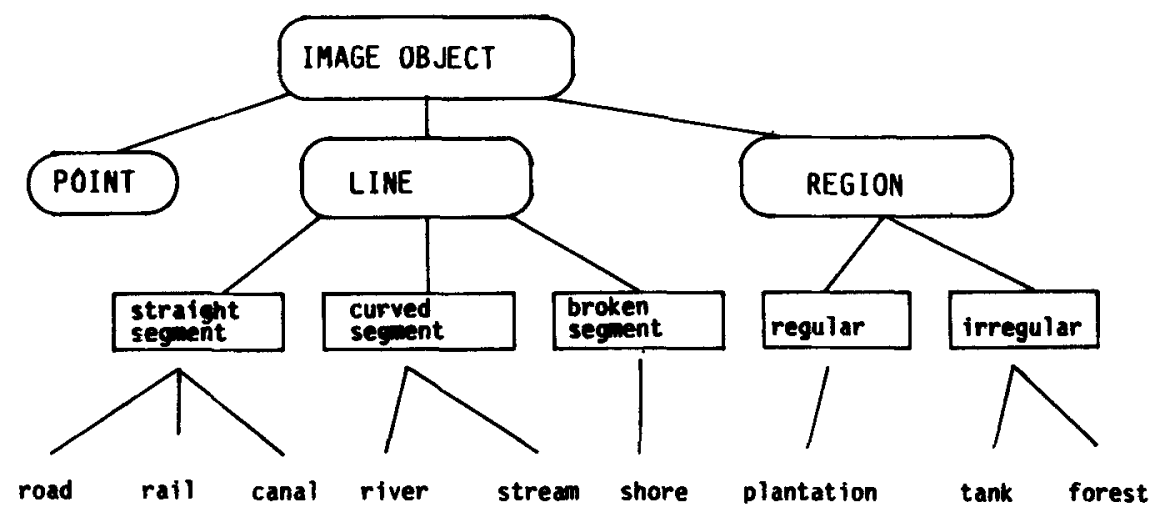

(c)

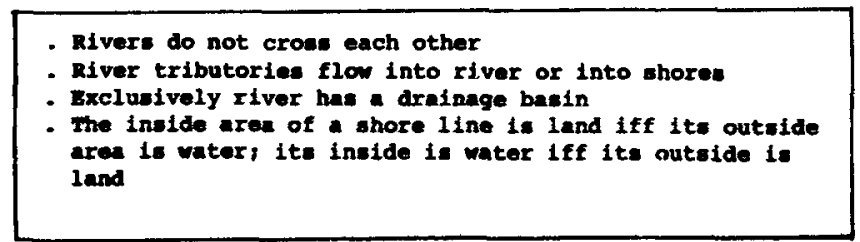

Figure 3. Image domain (a) and scene domain (b) specifications, and real-world knowledge (c).

The knowledge about a line and a point object is represented interpretation keywise in the form of rules as shown below.

Line object

(SETQ LINE(APPEND LINE'(RAIL ROAD RIVER CANAL)))

(SETQ RULE41'(COND (EQ COLOUR 'BLUISHGREEN))

(SETQ SETI'(ROAD))) ('T (SETQ SETI'NIL))))

Point object

(SETQ POINT (APPEND POINT'(INDUSTRIAL-SHED

BUILDING TREE SETTLEMENT))

(SETQ RULE(APPEND RULE(QUOTE(RULE 2)

(SETQ RULE2'(COND ((EQ COLOUR 'RED)

(SETQ SETI'(TREE))) ('T(SETQ SETI'NIL)) 
An object-oriented approach for knowledge representation as shown above provides an environment for modular software design. Because of run-time binding facility in AI programming language LISP, it is possible to store and modify object data, facts and rules dynamically across different types and classes of logical objects of an image. Property-list structures are helpful in extending the existing knowledge base for future updates. Default knowledge and assumptions are stored in the form of rules, which take care of side-effects of the reasoning mechanism. Thus knowledge representation requirements are met effectively with an object-oriented approach.

It is observed that the time taken for the identification process for region objects based on property lists as facts in the knowledge-base is significantly high. Hence it is decided to go for rules structure in the knowledge base for line/point objects though the overall computation time complexity is the same (Sarma 1991).

\section{Reasoning for identification}

Satellite image interpretation activity involves analysis of an image to decrease the local ambiguity by fusing the pieces of knowledge associated with the interpretation keys into a unique interpretation. For example, each interpretation key may suggest one or more land cover categories; crop land is identified by the colour signature of bright red to red whereas plantation (agriculture) is identified by the colour signature dark red to red. This indicates that there is an overlap in the description of signature and hence colour alone may indicate two categories. Hence, we consider the other interpretation keys such as texture and pattern and fuse the knowledge from them with colour signature and bring out consensus to identify crop land and plantation. Thus image interpretation problem can be seen as a data fusion activity, in the sense that individual elements of an image object have to be associated in order to produce a comprehensive and unique interpretation. This approach helps in removing brittleness in decision.

\subsection{Basic theory}

We have applied the Dempster-Shafer (D-S) theory of evidence to re,note sensing satellite image interpretation for combining of evidence associated with the interpretation keys for the identification of a target object on a given false colour composite. In this theory, the belief in a proposition $A$, is expressed by a subinterval $[s(A), p(A)]$ of the unit interval $[0,1]$. The lower value $s(A)$ represents the 'support' for that proposition and sets a minimum value for its likelihood. The upper value, $p(A)$, denotes the 'plausibility' of that proposition and establishes a maximum likelihood. 'Support' may be interpreted as the total positive effect a body of evidence has in a proposition, while 'plausibility' represents the total extent to which a body of evidence fails to refute a proposition. The degree of uncertainty about actual probability value for a proposition corresponds to the width of its interval.

\subsection{Formulation of representation of evidence}

Let $F$ be the mutually exclusive and exhaustive set of propositions in the domain, called frame of discernment or universe of discourse. Elements of the power set $2^{F}$, that is, subsets of $F$ are the class of general propositions in the domain. Let $N$ be 
the number of features/interpretation keys based on whose values an object (point, line or area) is identified. These interpretation keys are considered as knowledge sources $\{k s 1, k s 2, \ldots, k s n\}$.

\subsection{Dempster's rule of combination}

In this, each knowledge source distributes a unit belief across a set of propositions for which it has evidence. These propositions are referred to as focal elements of corresponding knowledge sources. The distribution is in proportion to the weight of that evidence as it bears on each proposition.

General formalism of the above description may be represented by a function

$$
m_{1}=\left\{A_{i} \mid A_{l} \subset F\right\} \rightarrow[0,1],
$$

where $F$ is a mutually exclusive and exhaustive set of propositions in the domain.

Support for a proposition.

$$
s_{1}(A)=\sum_{A_{i} \in A} m_{1}\left(A_{i}\right) .
$$

$s_{1}(A)$ is also denoted by $\operatorname{Bel}(A)$ which indicates total belief of $A . \operatorname{Bel}(A)$ is called a belief function if it satisfies the following properties ( $\mathrm{Ng} \&$ Abramson 1990):

(i) the belief in a null hypothesis is 0 ;

(ii) the belief in $F$ is 1 ;

(iii) the sum of beliefs of $A$ and $\tilde{A}$ must be less than or equal to 1 .

Total mass $m(C)$, combining masses from two sources $k s 1$ and $k s 2$ are combined using the formula (here $C$ is a given subset of $F$ )

where

$$
m(C)=\left[\begin{array}{l}
0 \text { if } C \text { is } \phi, \\
\sum_{A_{i} \cap B_{j}=C} \frac{m_{1}\left(A_{i}\right) \cdot m_{2}\left(B_{j}\right)}{1-k},
\end{array}\right.
$$

$$
k=\sum_{A_{k} \cap B_{l}=\phi} m_{1}\left(A_{k}\right) \cdot m_{2}\left(B_{l}\right)
$$

The resultant $m(C)$ is a new body of evidence representing the combination of two original bodies of evidence. The new evidence may in turn be combined with evidence from other sources. This is the process of belief of propagation in D-S theory (Garvey et al 1981).

\subsection{Suitability to remote sensing}

The suitability of the Dempster-Shafer theory for remote sensing is justified because of the following reasons.

(1) The combination rule tries to discard conflicts by way of normalization and brings out consensus.

(2) Order of combination is immaterial because of commutativity and associativity of multiplication which is the primitive operation in belief combination and propagation. 
(3) Dempster's combination rule acts over the entire subset space. Because of this, computations grow exponentially over the set of identification names i.e., $F$ (frame of discernment). But in remote sensing image interpretation, some subsets are not required to be taken into consideration, as for such subsets there will be no evidence since spectral signature alone is sufficient to label some objects.

(4) Ignorance of the user in apportioning his belief can be carried out till the end of processing in a structured way. With this facility, the user is not forced to label his belief to any one or a combination of identification names.

\subsection{Example of application of Dempster's combination rule}

Suppose we have some possible subsets of identification names that are contributing evidence as indicated in the figure 4.

Evidence 1 from feature, colour:

- Belief in vegetation $=0 \cdot 3$, belief in soil $=0.5$, not known (undistributed) $=0 \cdot 2$.

Evidence 2 from feature, texture:

- Belief in soil or water $=0.7$, not known (undistributed) $=0 \cdot 3$.

- Summed up value for vegetation $=0.09$, summed up value for soil or water $=0.14$, summed up value for soil $=0.35+0.15=0.5$, summed up value for undistributed $=0.06$, conflict (null set) $=\mathbf{0} \cdot \mathbf{2 1}$, pooled belief for vegetation $=0 \cdot 09 /(1-0 \cdot 21)=0 \cdot 11$, pooled belief for soil or water $=0 \cdot 14 / 0 \cdot 79=0 \cdot 18$, pooled belief for soil $=0.59 / 0.79=0.63$, uncertainty $=0.06 / 0 \cdot 79=0.08$, plausibility (soil) $=1-\operatorname{Bel}(\neg$ soil $)=1-\operatorname{Bel}($ vegetation $)=1-0 \cdot 11=0 \cdot 89$, evidential interval for soil is $[0.63,0.89]$, ignorance $=0.26$.

Interpretation of results:

- With the available evidence 'soil' is the identification name, considering maximum value of belief.

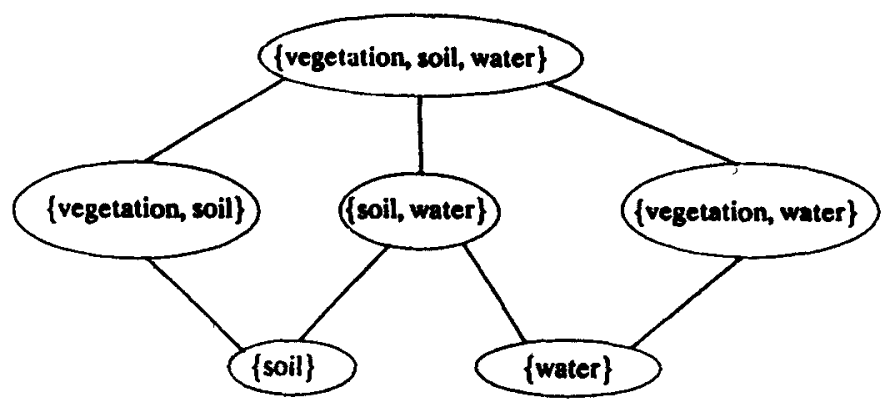

Figure 4. Sample subsets of a set of identification names contributing evidence. 


\section{Methodology}

We have bifurcated a given image into logical objects manually into the appropriate types such as point, line or region. Let $s[i]$ be the set of possible identification names representative of $i$ th feature value given by a user with his confidence value $c[i]$. Thus we have $N$ sets of $s[i]$ and $c[i]$ for $i=1,2, \ldots, N$. Let $m(s[i])$ denote the weightage (or a portion of belief) indicating that the identification name is in the subset $s[i]$ of $F$, frame of discernment. Assignment of this weightage is the crux of the problem and is the basis for getting successful results. Two methods are adopted to decide $m(s[i])$.

\subsection{Basic probability assignment}

Method 1: User's confidence in the description of interpretation key value is taken directly as $m[s(i)]$. This is analogous to the way an expert does, that is, totally depending on his confidence. With $m(s[i])$, weightage for a particular identification name is calculated by summing up the confidence values of sets in which the identification name occurs. Thus the name with maximum weightage is considered as the identification name. This method is termed "confidence".

Method 2: The idea of taking $c[i]$ as $m(s[i])$ directly may lead to a brittle decision because of the following. While inputting the confidence values, humans may not be consistent always. It is difficult to apportion belief in the same proportions always. In such situation, we feel that the cardinality of the subset $s$ [i] is to be taken into consideration and we have done so in calculating $m(s[i])$. Thus $m(s[i])$ is the product of $c[i]$ and $1 /|s[i]|$; here equal chance of occurrence of any name in the set $s[i]$ is the heuristic. This method is named "system". Dempster's combination rule is applied to these two methods.

\subsection{Interpretation rules}

The Dempster-Shafer approach provides specific numerical values of belief and plausibility allowing the residual uncertainty to exist. Interpretation of these values as qualitative results is to be done by the system designer. We have interpreted the results depending on following interpretation rules.

(1) Label the identification name having maximum plausibility and belief value compared to all others.

(2) If two labels have the same belief, then the one with the higher plausibility is considered. This is because the same belief does not mean the same plausibility.

(3) If two or more labels have the same belief and plausibility then suspend judgement and guide the user to go for collateral data.

(4) Set threshold values for belief, plausibility and evidential interval and judge the label name.

\subsection{Knowledge organization and control sequence}

Normally ordering of pattern features has a direct effect on the efficiency of recognition (Makato 1984). But in the D-S method it is immaterial because of associativity and commutativity of multiplication operation which is the key for combination and 
propagation of evidence. Our method is the bottom-up procedure in which we constructed the required evidence from the feature values along with user's confidence. A sequential method of control is used for the identification of category name of a given object on FCC. The sequence used is pattern feature colour, pattern, texture, shape, size, association, relief and season. Each step is a partial decision making step, precipitating the available evidence to formulate subcategories. Steps go on until a subcategory contains only one identification name or no more evidence accumulation is possible.

\section{Description of the system}

The functional flow diagram of the software system is shown in figure 5 . The software package is menu-driven having facilities to store facts and rules, to store image objects to be identified, to modify facts and rules and the inferencing mechanism to identify a target object. Explanation is provided at user's option and 'learning' is incorporated which uses its experience acquired based on the systems previous usage. Appropriate warnings and explanatory messages are given at the required places for an easy operation of the software. Summary of programs developed for construction of the knowledge bases and identification is given below.

\subsection{Construction of knowledge bases}

The knowledge base of the system consists of facts-base REGFAC.LSP, rule-base OBJRUL.LSP and learning sets LEARN.LSP occupying a storage space of $51 \mathrm{k}$ bytes. The knowledge base can be updated and modified as and when the new facts are collected. From the interactive session with user, the system itself chooses and forms

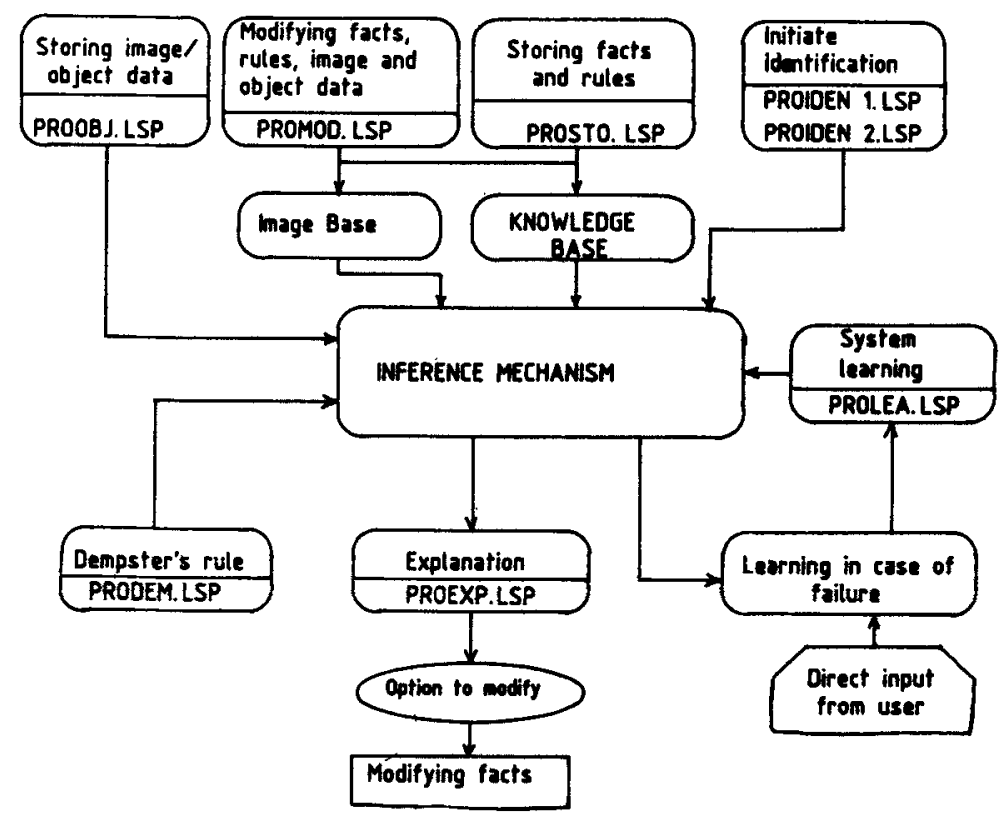

Figure 5. Functional flow diagram of the software. 
appropriate knowledge structure and stores in the knowledge base. Some of the lists maintained by the system's knowledge base are given below.

\section{(i) REGION/LINE/POINT}

This consists of a set of possible identification names on which facts/rules exist in knowledge base. The universe of discourse or frame of discernment is formed here.

(ii) RULE

This consists of the set of all rule name, RULEi, in the system. Also the facts in REGFAC.LSP are classified as class and subclass.

The data of logical image object to be identified on a given FCC is stored in the form of list in IMAGE.LSP. This the maintains two lists namely, IMAGE containing FCC imagery $I i$ and OBJECT containing the set of all objects, $O i$, pertaining to $I j$.

\subsection{Identification of image objects}

The identification mechanism is initiated by the files PROIDEN1.LSP and PROIDEN2.LSP. These files have 26 functions constituting the inference mechanism for identifying a target object on FCC whose data is stored in IMAGE.LSP. Also they call functions in PRODEM.LSP, PROLEA.LSP and PROEXP.LSP according to the options exercised by user.

Once the object $O i$ on an FCC is chosen, based on the type of object, namely region or line or point, the respective expert, namely, REGEXP/LINEXP/POIEXP is triggered. These experts make use of facts and rules in knowledge-base (REGFAC.LSP and OBJRUL.LSP) and lead to formation of ten sets $S 1$ to $S 10$, one for each feature, which contain the possible identification names based on the match between the corresponding feature value of the object to be identified and that of identification name. List of confidence values entered by the user for each feature of the object to be identified is formed in CONF. List of pairs (sub-expert, rules fired) is stored in RESUL1. Having formed the above mentioned lists and sets, the function INFER of PROIDEN2.LSP is executed, which gives the user the options of methods of identification of the object chosen as shown in figure 6. For DEMPSTER method, functions in file PRODEM.LSP are made use of. If the user wishes to use the learning done by the system previously, learning sets in LEARN.LSP are made use of.

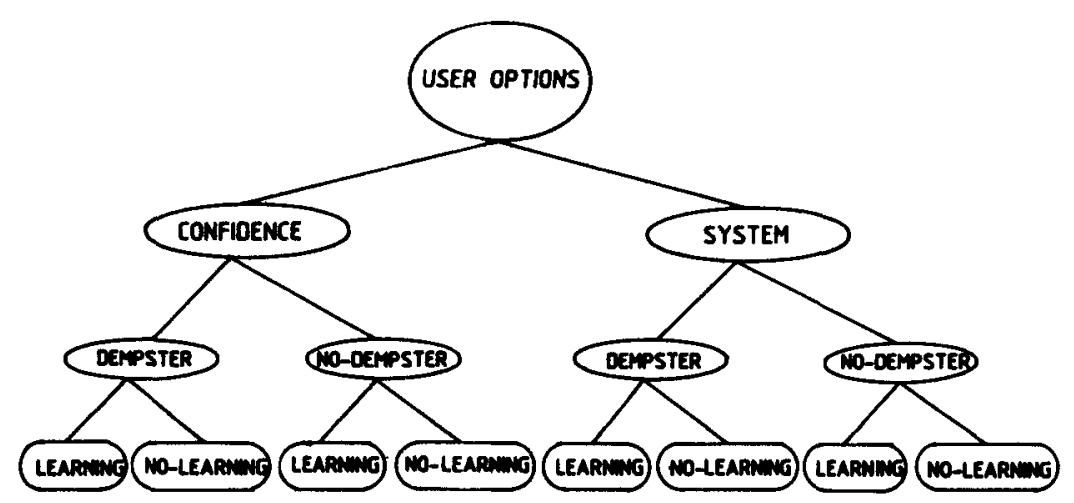

Figure 6. Options in the method of image-object identification. 


\subsection{Explanation}

Various intermediate results and procedural explanation for each method of identification selected, can be seen during explanation session, which makes use of file PROEXP.LSP. Explanation facility is provided by tracing the rules fired in the sequence and giving the plausibility and belief values for the set in which the identified object is a member.

\subsection{Learning}

In case the result arrived is an incorrect one, the user can give the correct answer so that the system can reallocate the weightages to each of the sets Si during identification to arrive at the correct solution. Sometimes, learning may fail if all the sets"Si containing the correct answer, also contain the incorrect answer in which case the user is prompted with an appropriate message.

\subsection{Computation complexity}

Dempster's combination rule acts over the entire subset space of frame of discernment, i.e. the set of identification names. Hence identification by the Dempster-Shafer approach has computation complexity of the order $O\left(k \cdot n \cdot 2^{n}+c\right)$, where $n$ is the cardinality of frame of discernment and $k$ and $c$ are constants.

\section{Results of identification}

The knowledge base of the system has been developed and tested on the basis of three FCC of IRS-1A in addition to a hypothetical test image. In this paper we present the details of one FCC (shown in figure 7) and the hypothetical test image. Some of the detectable image-objects are indicated on the images by decimal numbers. The features of these objects are extracted upon consultation with a skilled interpreter.

The results of identification of the image-objects, namely 019 to 023 , are shown in figure 8. Objects $O 1$ to $O 4$ belong to the hypothetical image $I 1$ and are considered for purpose of testing. Objects $O 19$ to $O 23$ belong to the image shown in figure 7 and are tagged to a symbolic image name $I 4$ in the knowledge-based system. Objects $O 5$ to $O 14$ belong to a symbolic image $I 2$ and objects $O 15$ to $O 18$ belong to $I 3$. For details on the objects $O 5$ to $O 18$ the reader is referred to Sarma (1991). Results are obtained exercising all the 8 options as shown in figure 6 . Each user option is a path from the root to a leaf node. The results are compared with an expert's opinion as shown in the last column of figure 8 . Results are correct to the extent of $95 \%$ in land use/land cover domain (cnastal belts) for which we have developed the knowledge base.

\subsection{Critical evaluation}

From the results obtained it is observed that SYSTEM measure of obtaining $m(S[i])$ is more accurate than CONFIDENCE measure. The $D-S$ approach of identification with SYSTEM measure is more accurate, and it also helps in analysing the results with respect to plausibility, belief and evidential interval. It is appropriate to highlight that in case of object $O 2$, with options CONFIDENCE and NODEMPSTER, learning may 


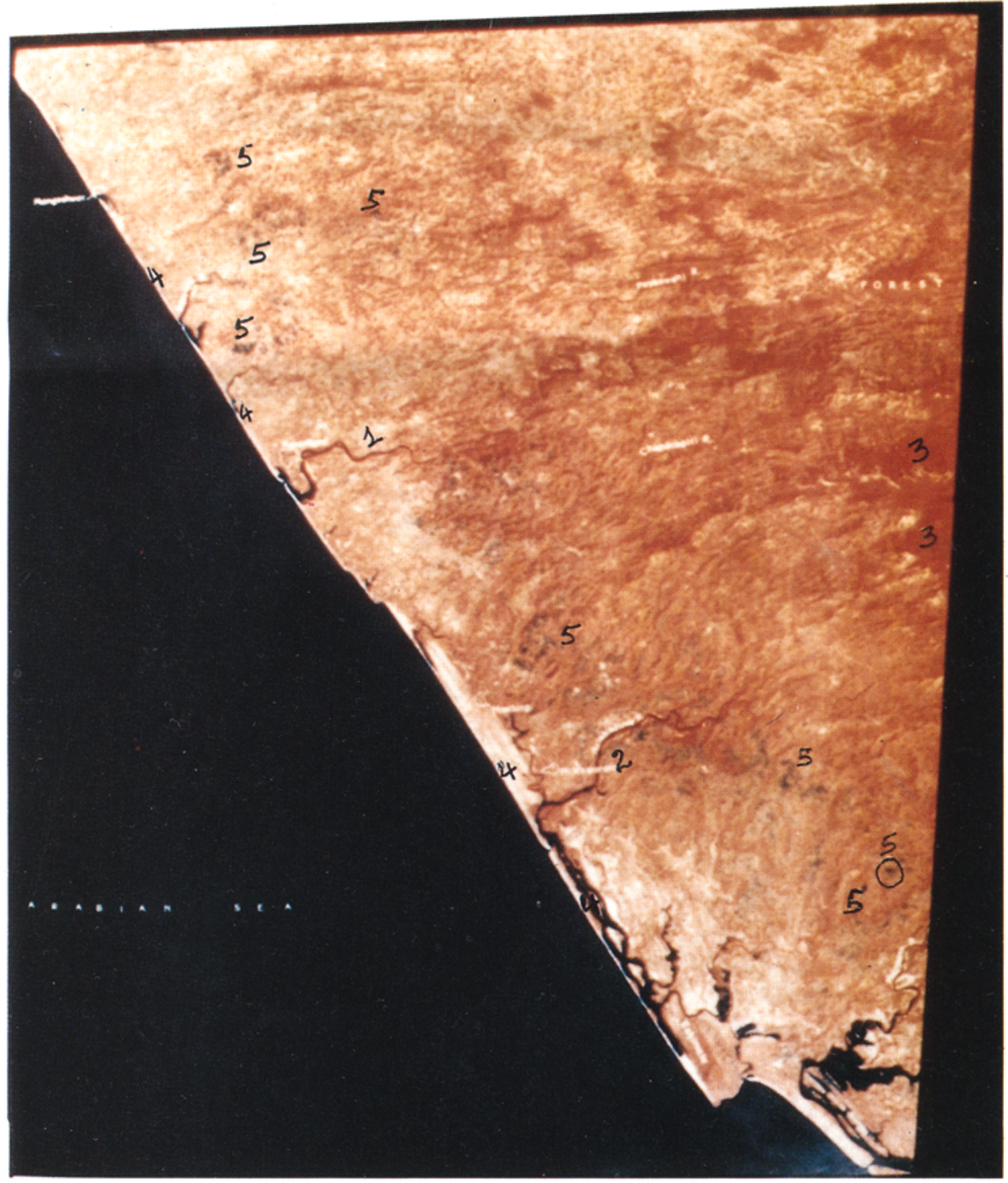

Figure 7. FCC under interpretation.

fail, assuming the correct identification is $R 3$ (fallow land). It is due to the fact that $R 3$ occurs in the set $(R 3, R 14, R 28)$, and is an incorrect answer. So giving higher weight to sets $S i$ containing $R 3$ would ultimately result in increasing the weightage for the incorrect result of identification also.

It is evident that the result of identification of object $O 21$ with options CONFIDENCE and DEMPSTER with No-learning is wrong, that is, $O 21$ has been identified as crop land $(R 2)$ instead of evergreen forest $(R 6)$. Further the system has been provided with the correct answer $R 6$ during the learning session. The rock exposures (laterite cappings) on the image can be seen as point objects spread almost throughout the coastal side of the scene. These are identifiable because of the significant feature, the 


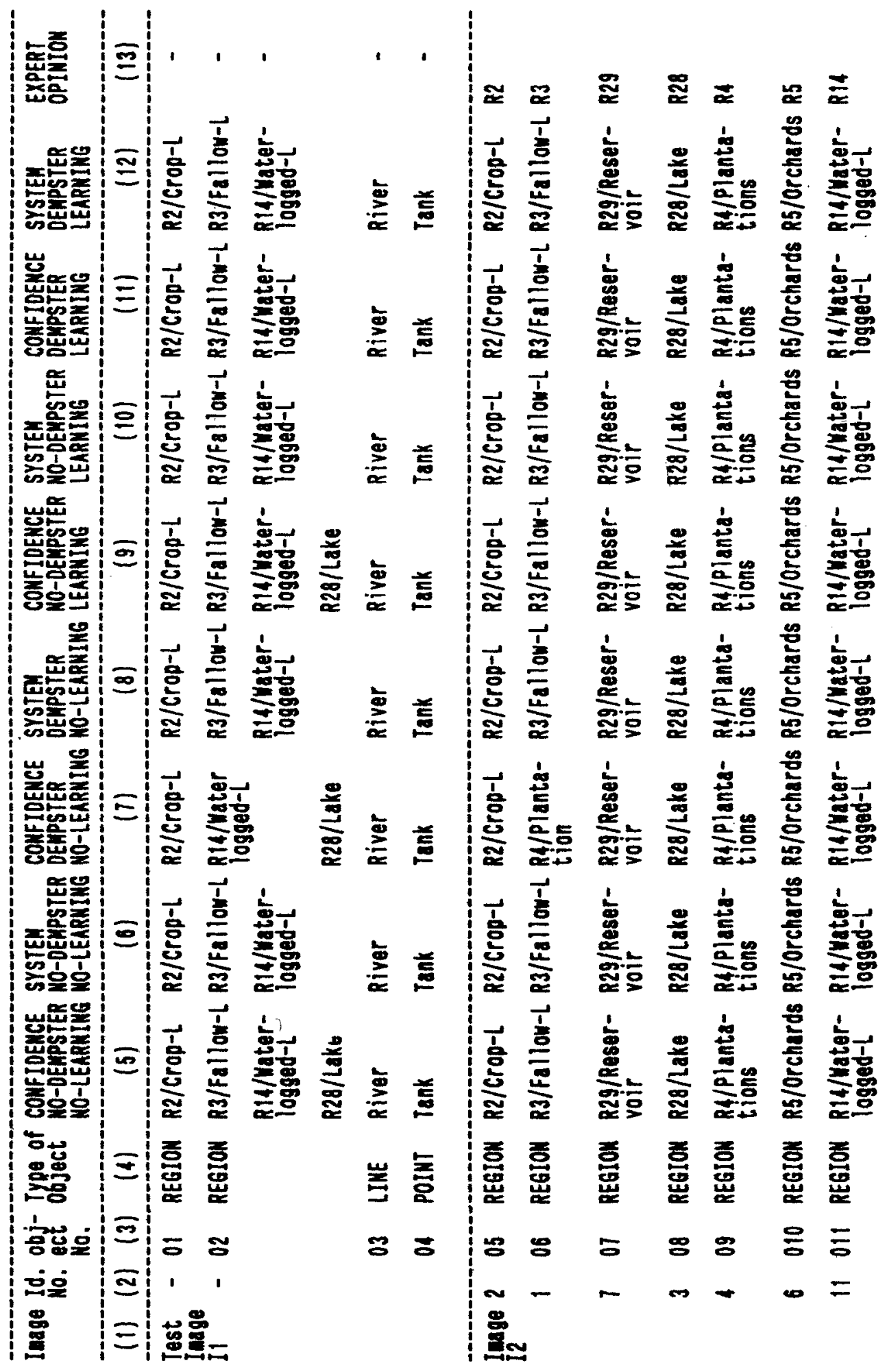




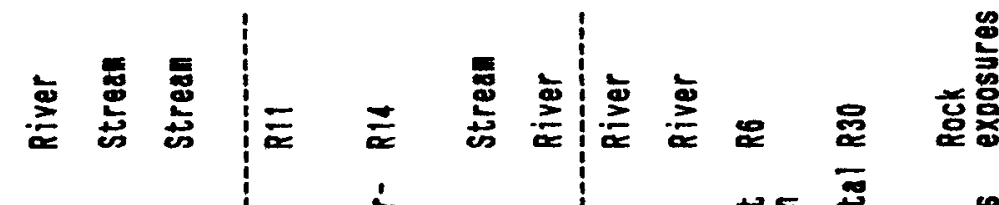

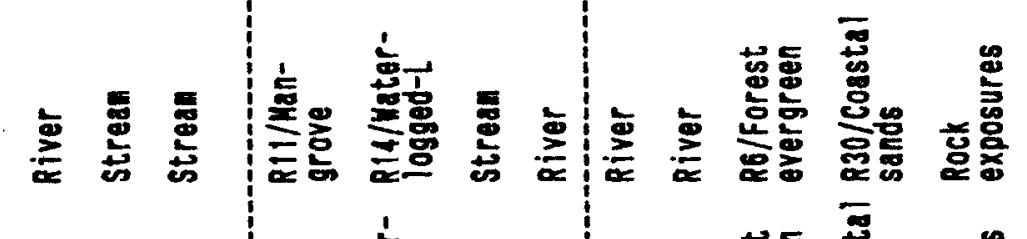

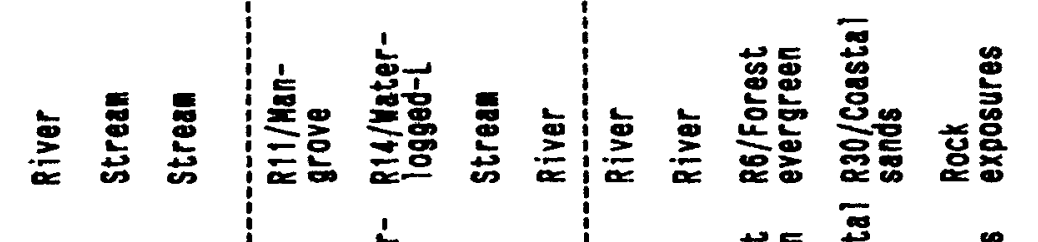

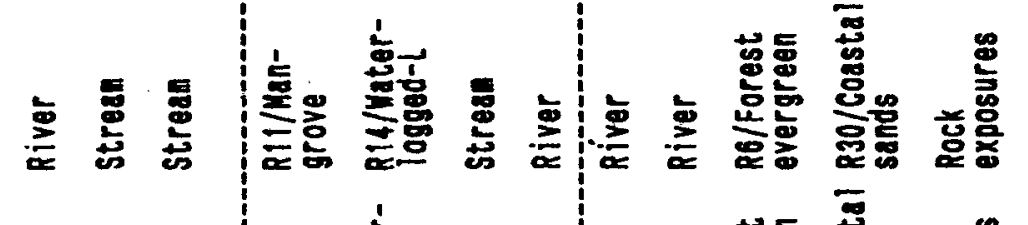

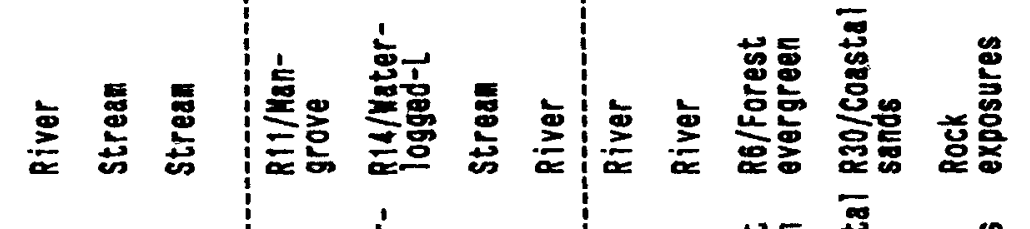

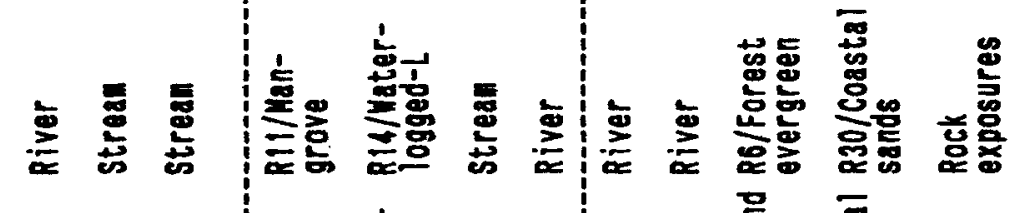

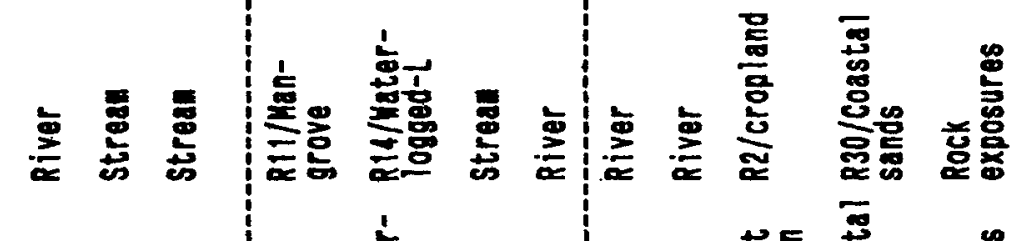

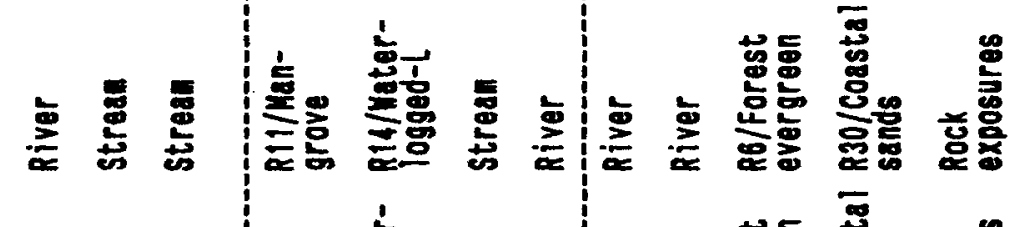

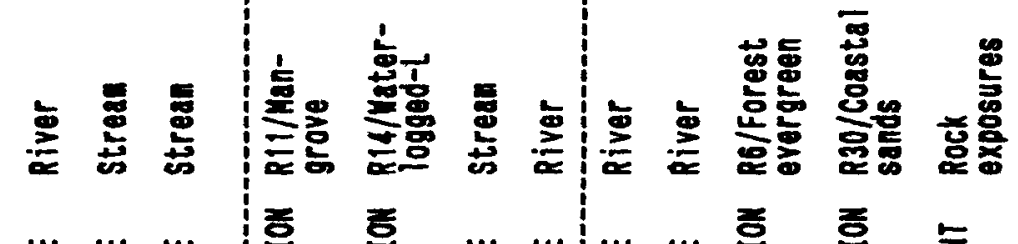

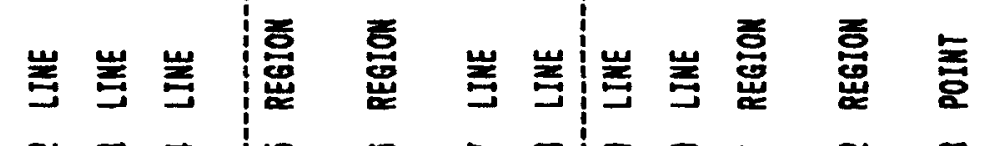
亏

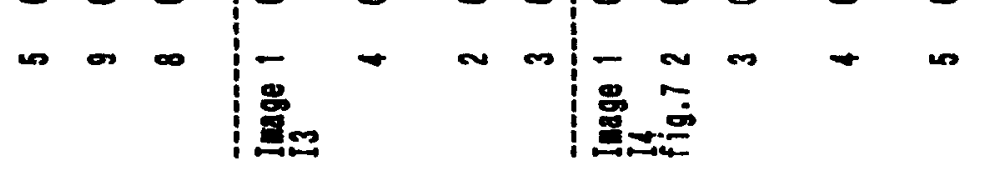


relief of undulating low hills devoid of vegetation. From the photograph in figure 7 , the relief may not be striking. However, in the original image it is apparent.

\section{Conclusions}

In this paper, we have demonstrated the application of knowledge-based methods for remote sensing satellite image interpretation. Our motivating assumption, that image interpretation is a form of intelligence-computation involving qualitative reasoning, is realized in the process of development of the prototype expert system. We have considered two basic probability assignment methods, namely 'confidence' and 'system' and combined each one with (or without) Dempster's combination rule (with or without learning). Thus 8 options have arisen for identification. We have carried out the identification process exercising all these options in a bid to analyse the consistency and correctness of the methods and found that the 'system' method is more accurate than the 'confidence' method. For image objects which have similar features, threshold values for plausibility, belief and evidential intervals are critical for correct identification. It is observed that the time taken to reason with regard to objects represented in property lists is significantly large. So we have decided to maintain the knowledge of line and point objects in the form of rules, leaving region objects' data in property lists. This has improved the speed of execution though the overall computation complexity is same.

Although we have taken the standard FCC of IRS-1A for identification and analysis of results, use of the system is not restricted to FCC only. It can also be used to interpret black-and-white images or any other photographic data products for which experts can design an interpretation key. Since the system has taken the shape of an expert system shell, removing the existing knowledge base and providing a new knowledge base would enable it to be used for the interpretation tasks described above. At present, our system may be used as an aid to an expert interpreter.

\section{References}

Argialas P D 1990 Computational image interpretation models: An overview and perspective. Photogrammetric Eng. Remote Sensing 56(6): 871-876

Garvey T D, Lowrance J D, Fischler M A 1981 An inference technique for integrating knowledge from disparate sources. International Joint Conference on Artificial Intelligence 1981 Proceeding: 319-325

Hayes-Roth F 1989 Towards benchmarks for knowledge systems and their implications for data engineering. IEEE Trans. Knowledge Data Eng. 1(1): 101-110

Makato N 1984 Control strategies in pattern analysis. Pattern Recogn. 17(1): 45-56

Ng K C, Abramson B 1990 Uncertainty management in expert systems. IEEE Expert: 29-47, April 1990

Sarma L C 1991 Knowledge based interpretation of remote sensing data. M Sc (Eng.) Thesis, Department of Computer Science and Automation, Indian Institute of Science, Bangalore

Sarma L C, Sarma V V S 1990 A knowledge based system for land use/land cover categorization. Proceedings of Artificial Intelligence and Expert System Technologies in the Indian Context, Tata McGraw-Hill Publishing Company Ltd., Volume 2: 108-114

Schowengerdt R, Wang H 1989 A general purpose expert system for image processing, Photogrammetric Eng. Remote Sensing 55: 1277-1284

Wang F, Newkirk R 1988 A knowledge based system for highway network extraction. IEEE Trans. Geosci. Earth Sci. 26(5): 525-531

Wharton W S 1987 A spectral knowledge-based approach for urban land cover discrimination. IEEE Trans. Geo-Sci. Remote Sensing 25: 272-282 